\title{
Subwavelength Localization of Atomic Excitation Using Electromagnetically Induced Transparency
}

\author{
J. A. Miles, Z. J. Simmons, and D. D. Yavuz \\ Department of Physics, 1150 University Avenue, University of Wisconsin at Madison, Madison, Wisconsin, 53706, USA
}

(Received 19 April 2013; revised manuscript received 24 June 2013; published 11 September 2013)

\begin{abstract}
We report an experiment in which an atomic excitation is localized to a spatial width that is a factor of 8 smaller than the wavelength of the incident light. The experiment utilizes the sensitivity of the dark state of electromagnetically induced transparency (EIT) to the intensity of the coupling laser beam. A standing-wave coupling laser with a sinusoidally varying intensity yields tightly confined Raman excitations during the EIT process. The excitations, located near the nodes of the intensity profile, have a width of $100 \mathrm{~nm}$. The experiment is performed using ultracold ${ }^{87} \mathrm{Rb}$ atoms trapped in an optical dipole trap, and atomic localization is achieved with EIT pulses that are approximately $100 \mathrm{~ns}$ long. To probe subwavelength atom localization, we have developed a technique that can measure the width of the atomic excitations with nanometer spatial resolution.
\end{abstract}

\section{INTRODUCTION}

It is well known that traditional optical techniques cannot resolve or write features smaller than half the wavelength of light. This barrier, known as the diffraction limit, has important implications for a wide variety of research areas that utilize optical tools. For example, several architectures for quantum computing, such as those using trapped ions or trapped neutral atoms, use focused laser beams to initialize and manipulate qubits [1-5]. The diffraction limit prohibits high-fidelity manipulation of individual qubits separated by less than half the wavelength of the excitation light. As a result, the distance between qubits must be larger than the wavelength, but this limits the twoqubit interaction (for example, a Rydberg dipole-dipole interaction for neutral atoms). The necessary qubit spacing, in turn, limits the fidelity and also puts an upper bound on the speed of two-qubit gates. A fast and reliable technique to address and manipulate qubits with nanometer resolution will have significant implications for quantum computing. In this work, we report an important step towards this goal. We use the dark state of electromagnetically induced transparency (EIT) [6-9] and demonstrate the localization of hyperfine excitation to a spatial width of $100 \mathrm{~nm}$, which is a factor of 8 smaller than the wavelength of the EIT beams. We achieve this localization using 100-ns-long EIT pulses, i.e., with a gate speed capability of about $10 \mathrm{MHz}$.

Before proceeding further, we note that there are other proposed techniques that achieve spatial localization of atomic excitation beyond the diffraction limit. The use of

Published by the American Physical Society under the terms of the Creative Commons Attribution 3.0 License. Further distribution of this work must maintain attribution to the author(s) and the published article's title, journal citation, and DOI. the dark state for nanoscale-level qubit-addressing has the following key advantages: (i) For laser pulses varying slowly compared to their instantaneous Rabi frequency, the dark-state technique results in negligible population in the excited electronic state. As a result, nanoscale resolution can be achieved without suffering from the detrimental effects of spontaneous emission. (ii) The dark state can be prepared adiabatically, and thus the scheme is insensitive to many experimental fluctuations such as driving laser-pulse intensity and timing variations. (iii) Since the scheme is coherent, with sufficiently intense laser beams, qubit manipulation can be achieved at fast (nanosecond) time scales with high fidelity. Because of these advantages, the dark-state approach is particularly well suited for quantum-computing applications, where fast and high-fidelity coherent manipulations with little decoherence are required.

\section{A. Electromagnetically induced transparency and the dark state}

EIT is a technique that uses quantum interference to eliminate the absorption of a resonant laser rendering a medium transparent [6-9]. As shown in Fig. 1(a), we consider a three-level $\Lambda$ system interacting with two laser beams, the probe and coupling lasers, with Rabi frequencies $\Omega_{P}$ and $\Omega_{C}$, respectively. Without the coupling laser, the probe beam, which is resonant with the $|1\rangle \rightarrow|e\rangle$ transition, will be absorbed by the medium. With the coupling laser, however, a transmission window whose width is determined by the intensity of the coupling beam is established in the medium. EIT occurs because, in the presence of the two laser beams, the atoms are driven to a dark state with negligible population of the excited level $|e\rangle$. As a result, the atoms become completely decoupled from the two lasers, and the medium becomes transparent to both laser beams. 
(a)

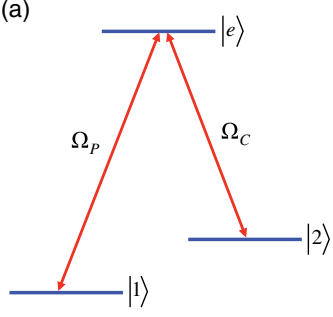

(b)

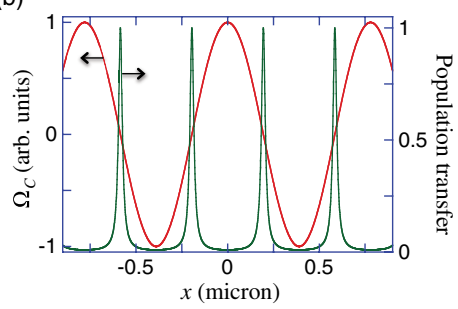

FIG. 1. (a) Three-level $\Lambda$ scheme for driving the atoms to a dark state and observing EIT. With atoms starting in the ground state $|1\rangle$, the dark state can be prepared using a counterintuitive pulse sequence; i.e., $\Omega_{C}$ should be turned on before $\Omega_{P}$. (b) Spatial localization of excitation using the dark state. Because of the sinusoidal intensity profile of the coupling laser (standing wave), the population transfer to state $|2\rangle$ is localized to regions near the intensity nodes. The width of the localized regions is controlled by the ratio of the intensities of the probe and coupling laser beams.

The most straightforward approach for preparing the atoms into the dark state starting from the ground state $|1\rangle$ is to use a counterintuitive pulse sequence; i.e., the coupling laser should be turned on before the probe laser beam. In the ideal limit, the dark state does not have any component in state $|e\rangle$, and it is given by

$\left|\psi_{\text {dark }}\right\rangle=\frac{\Omega_{C}^{*}}{\sqrt{\left|\Omega_{P}\right|^{2}+\left|\Omega_{C}\right|^{2}}}|1\rangle-\frac{\Omega_{P}^{*}}{\sqrt{\left|\Omega_{P}\right|^{2}+\left|\Omega_{C}\right|^{2}}}|2\rangle$.

In addition to its prominent role in EIT, the dark state of Eq. (1) is central to a number of effects such as coherent population trapping $(C P T)$ and stimulated Raman adiabatic passage (STIRAP) [10]. Over the last decade, there have been a number of exciting advances that utilize EIT and these related techniques. Of particular importance, it is now understood that, using these techniques, one can obtain slow light [11-16] and stopped light [17-22], and also construct optical nonlinearities that are large enough to be effective at the single-photon level [23-28].

\section{B. Atomic localization using the dark state}

Recently, it has been suggested that the dark state can also be utilized to localize an atomic excitation to nanometer spatial scales. This approach was first proposed by Agarwal and colleagues [29] and was later expanded by us [30] and the Lukin group [31]. The first evidence of darkstate-based localization was experimentally demonstrated in a vapor cell by Scully and colleagues [32]. We have recently reported a proof-of-principle experiment, where we observed such localization over large (sub- $\mathrm{mm}$ ) spatial scales using ultracold ${ }^{87} \mathrm{Rb}$ atoms trapped in a magnetooptical trap (MOT) [33]. In this approach, the key idea is to use the spatial sensitivity of the dark state to the intensity of the coupling laser beam. From Eq. (1), the population of state $|2\rangle$ is $\left|\left\langle 2 \mid \psi_{\text {dark }}\right\rangle\right|^{2}=\left|\Omega_{P}\right|^{2} /\left(\left|\Omega_{P}\right|^{2}+\left|\Omega_{C}\right|^{2}\right)$, which gets larger as the ratio $\left|\Omega_{C} / \Omega_{P}\right|$ decreases. This suggests

that in a region where the coupling laser goes through an intensity minimum, the population of state $|2\rangle$ can be very tightly localized. As discussed in Refs. [29,31], a simple approach to implementing this would be to use a coupling laser beam with a standing-wave spatial profile. For example, Fig. 1(b) shows the standing-wave field profile for a coupling laser that is resonant with the $\mathrm{D}_{2}$ line transition in $\mathrm{Rb}$ at a wavelength of $780 \mathrm{~nm}$. If the peak intensity of the coupling laser is much larger than the probe intensity, then only in localized regions of the couplingbeam standing wave is $\left|\Omega_{C} / \Omega_{P}\right|$ small. Therefore, only in these regions, which can be localized to spatial scales much smaller than the wavelength of light, is there significant excitation to state $|2\rangle$. It can be shown that, for a spatially uniform probe laser, the full width at half maximum of the excitation will be FWHM $_{\text {excitation }} \approx$ $\lambda\left(\Omega_{P} / \Omega_{C \text {, max }}\right)$, where $\Omega_{C \text {, max }}$ is the peak Rabi frequency of the coupling laser. Note that this expression can also be written in terms of the intensities of the respective beams, FWHM $_{\text {excitation }} \approx \lambda \sqrt{I_{P} / I_{C \text {, max }}}$ (assuming comparable dipole-matrix elements for the two transitions). As discussed in detail in Ref. [31], the localized population of level $|2\rangle$ can then be used to perform single-qubit or twoqubit gates with nanoscale spatial resolution. The standingwave intensity profile of Fig. 1(b) produces a periodic pattern of tightly localized regions. If a single localized spot is required, it can be obtained using a focused coupling laser beam with an intensity node at the center [30].

Before continuing, we note that a number of other approaches with similar goals of addressing atoms with subwavelength resolution have been proposed. In their pioneering work, Thomas and colleagues have suggested and experimentally demonstrated subwavelength position localization of atoms using spatially varying energy shifts [34-37]. Zubairy and co-workers have discussed atom localization using resonance fluorescence and phase and amplitude control of the absorption spectrum [38-43]. Knight and colleagues explored localization via quantum interference at the probability amplitude of the excited electronic state [44]. Various other schemes such as measurement-induced diffraction have also been discussed [45-49], and similar tools have been proposed for nanolithography using atomic beams [50]. There has also been remarkable progress in utilizing position-dependent stimulated emission to achieve nanoscale imaging resolution [51,52]. This last approach, also known as stimulatedemission depletion (STED) microscopy, is now a widely used technique in biological imaging. Although these are exciting developments, as explained above, the speed (nanosecond time scales) and suppression of spontaneous emission make the dark-state technique particularly attractive for quantum computing applications. Viscor et al. have recently investigated single-site addressing of individual atoms in a lattice with subwavelength resolution using the dark state of STIRAP [53]. Subwavelength optical lattices 
using the dark state have been discussed by Evers and colleagues [54]. Kiffner et al. have discussed enhancement in lithographic resolution using the generalized dark state of multiple cascaded $\Lambda$ schemes [55].

\section{EXPERIMENTS}

\section{A. Experimental schematic and EIT in a dipole trap}

We perform our experiments inside a 14-port stainlesssteel ultrahigh vacuum chamber that is kept at a base pressure of $5 \times 10^{-9}$ torr. A top view of our chamber is shown in Fig. 2(a). We start the experiment by cooling and loading the atoms into a MOT. To construct the ${ }^{87} \mathrm{Rb}$ MOT, we use three counterpropagating beam pairs that are locked to the cycling transition in the $\mathrm{D}_{2}$ line, each with a beam power of about $50 \mathrm{~mW}$ and a beam size of $3 \mathrm{~cm}$. A photograph of the MOT taken with an electron-multiplying CCD camera (EMCCD) is shown in Fig. 2(b). We typically trap about $1 \times 10^{9}{ }^{87} \mathrm{Rb}$ atoms at a temperature of $150 \mu \mathrm{K}$. To form the dipole trap, we focus a far-off resonant 850-nm laser beam to a spot size of $10 \mu \mathrm{m}$, overlapping it with the MOT cloud. The dipole-trapping beam comes from a diode-laser tapered amplifier system that produces an optical power up to $1 \mathrm{~W}$. Figure 2(c) shows a fluorescence image of the atoms trapped in the dipole trap. As we will discuss below, for the localization experiment protocol, it is important to have as low of an atomic temperature as possible. For this purpose, after the atoms are captured in the dipole trap, we adiabatically lower the trap depth to $50 \mu \mathrm{K}$ over a duration of $50 \mathrm{~ms}$. As a result, the atoms are evaporatively cooled to a temperature of $5.5 \mu \mathrm{K}$. We measure the atomic temperature by releasing the atoms from the dipole trap and recording the expansion of the atomic cloud.

The EIT beams are derived from a master externalcavity diode laser followed by high-frequency acoustooptic modulators and semiconductor tapered amplifiers. Further details of our laser system can be found in our previous publications [56,57]. As shown in Fig. 3(a), we set up an EIT $\Lambda$ scheme in ${ }^{87} \mathrm{Rb}$ using $F=1 \rightarrow F^{\prime}=2$ and $F=2 \rightarrow F^{\prime}=2$ transitions in the $\mathrm{D}_{2}$ line at a wavelength of $780 \mathrm{~nm}$, and we address these transitions with the probe and the coupling laser beams, respectively. The atoms are initially pumped to the $F=1$ level, and the experiment works in three parallel $m_{F}$ channels. As shown in Fig. 2(a), we form the coupling-laser standing wave by using a counterpropagating beam pair whose axis is at a slight angle to the propagation direction of the dipoletrapping beam. The probe laser propagates at a slight angle to both the dipole-trap and the coupling laser beams. Figure 3(b) shows the transmission as a function of frequency for a weak (optical power of about $1 \mathrm{pW}$ ) focused probe laser through the atoms in the dipole trap. The solid line is a fit to the data using the well-known EIT line shape. A transmission window is established in the cloud with a frequency width of $0.5 \mathrm{MHz}$, which is much smaller than the natural linewidth of the excited level $(\Gamma=6 \mathrm{MHz})$. These data are taken using only one of the coupling laser beams (i.e., there is not a standing-wave pattern) and with $200-\mu$ s-long EIT pulses. (a)

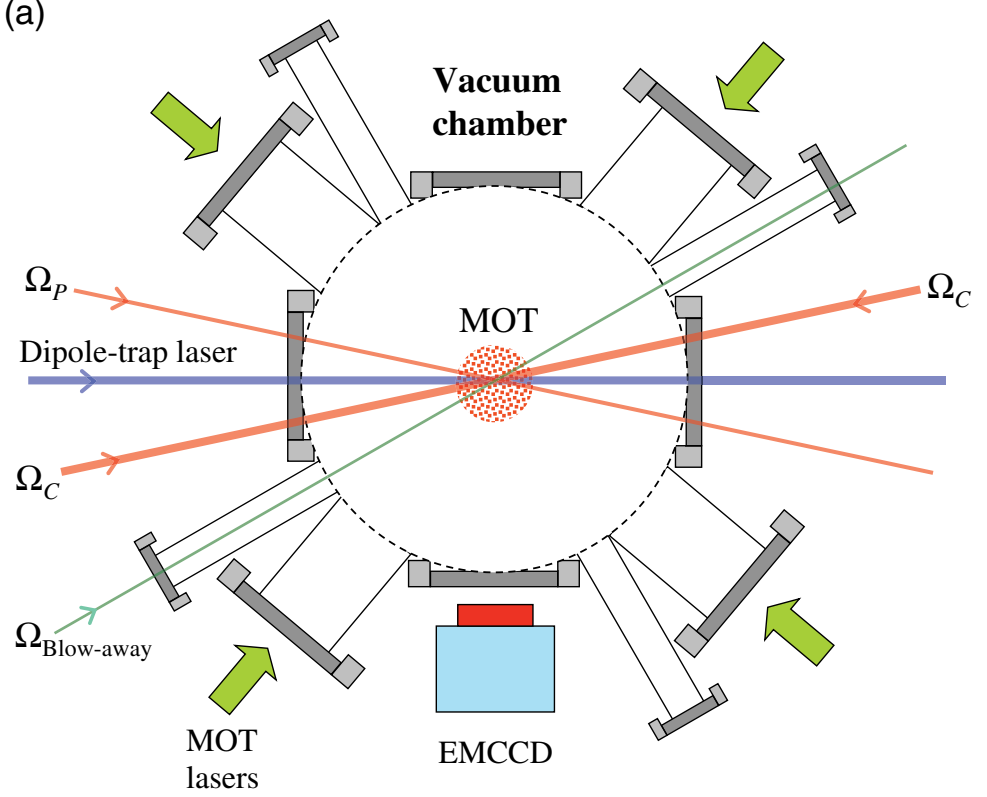

(b)

Photograph of our MOT

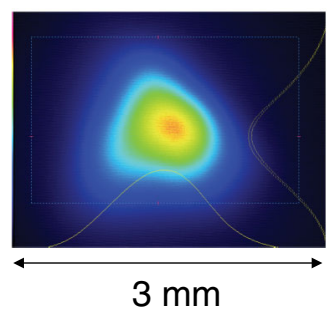

(c)

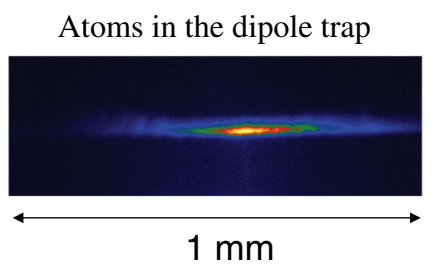

FIG. 2. (a) The top view of our ultrahigh vacuum chamber. The probe and the coupling laser beams propagate at a slight angle to the dipole-trapping beam direction. The coupling laser standing wave is formed using a counterpropagating beam pair. The blow-away beam, $\Omega_{\text {blow-away }}$, removes from the trap the atoms that have been transferred to the $F=2$ level. This beam is required for the localization measurement protocol as described in the text. (b) A photograph of the atoms trapped in the MOT. (c) The fluorescence image of the atoms trapped in the dipole trap. 
(a)

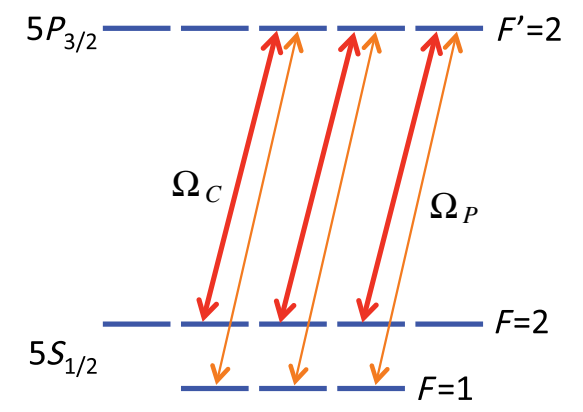

(b)

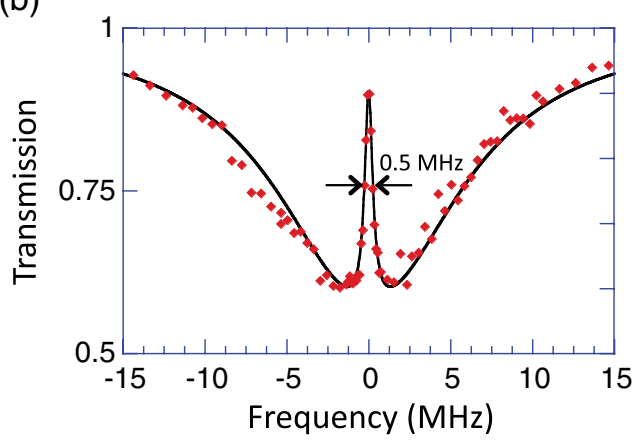

FIG. 3. (a) Energy-level schematic. We set up an EIT $\Lambda$ scheme in ${ }^{87} \mathrm{Rb}$ using $F=1 \rightarrow F^{\prime}=2$ and $F=2 \rightarrow F^{\prime}=2$ transitions in the $\mathrm{D}_{2}$ line, and we address these transitions with the probe and coupling laser beams, respectively. We start the experiment by pumping atoms to the $F=1$ level, and the experiment works in three parallel $m_{F}$ channels. (b) The transmission of a weak focused probe laser through the atoms in the dipole trap as a function of frequency. We observe good EIT, with a transparency width of $0.5 \mathrm{MHz}$.

\section{B. Coherent transfer with $\approx \mathbf{1 0 0 - n s - l o n g ~ E I T ~ p u l s e s ~}$}

We perform the localization experiments with probe and coupling laser beams of duration $50 \mathrm{~ns}$ and $100 \mathrm{~ns}$, respectively. For these experiments, the EIT beams are collimated in the chamber with a Gaussian $1 / \mathrm{e}^{2}$ intensity radii of $1.1 \mathrm{~mm}$ (probe) and $0.6 \mathrm{~mm}$ (coupling). We have experimentally determined and numerically confirmed that the localization experiments work best when the EIT beams are detuned by approximately $35 \mathrm{MHz}$ from the excited $F^{\prime}=2$ level. We work at a probe laser power of $5 \mathrm{~mW}$, which corresponds to a peak probe laser intensity of $I_{P}=260 \mathrm{~mW} / \mathrm{cm}^{2}$. Under these conditions, with the atoms initially pumped to the $F=1$ level, we find that the population transfer to the $F=2$ level is maximized for coupling laser beam power of $3 \mathrm{~mW}$ (which corresponds to a peak coupling laser intensity of $530 \mathrm{~mW} / \mathrm{cm}^{2}$ ). The optimal transfer near a coupling laser power of $3 \mathrm{~mW}$ is in agreement with our numerical calculations. Coupling powers lower than $3 \mathrm{~mW}$ result in imperfect EIT and, therefore, reduced transfer. Higher coupling powers reduce the transfer efficiency as suggested by the dark-state solution of Eq. (1). These investigations are performed using a single coupling laser, with each beam in the counterpropagating pair producing almost identical results.

To confirm the coherent nature of the transfer for excitation with such short pulses, we have performed a STIRAP experiment (again with a single coupling laser beam). Here, we set the power values of the probe and control lasers to $5 \mathrm{~mW}$ and $3 \mathrm{~mW}$, respectively, and record the population transfer to $F=2$ as a function of the time delay between the two pulses. As shown in Fig. 4, maximum transfer of $70 \%$ happens when the two laser pulses overlap such that both beams turn off simultaneously. In these data, this corresponds to the zero time delay point. This also means that the control beam is turned on $50 \mathrm{~ns}$ before the probe laser. We perform the localization experiments that will be detailed below at this zero time delay point.
The solid line in Fig. 4 is the result of simulations in which we solve the density matrix equations numerically for a single EIT channel with states $\left|F=1, m_{F}=0\right\rangle$, $\left|F=2, m_{F}=0\right\rangle$, and $\left|F^{\prime}=2, m_{F^{\prime}}=1\right\rangle$. When we use the calculated intensity values for both of the laser beams, we observe a slight mismatch in the flat regions of the STIRAP curve (nonoverlapping pulses) between the data and the simulation. We believe this mismatch is likely caused by a slight misalignment of the centers of the EIT beams from the atoms in the trap. We use this slight misalignment as an adjustable parameter to get a good fit between the experimental data and the simulation in the flat regions of the STIRAP curve. Aside from this adjustment, there are no further adjustable parameters in the simulation. As shown in Fig. 4, there is reasonable agreement between the simulation and the experimental data.

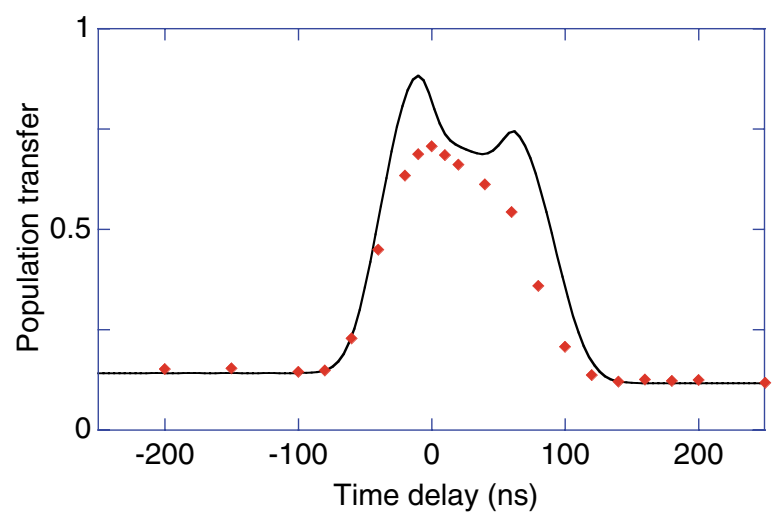

FIG. 4. The population transfer to the $F=2$ level (i.e., $|\langle\psi \mid F=2\rangle|^{2}$ ) as a function of the time delay between the probe and the coupling laser beams. The zero time delay point corresponds to overlapping the probe and coupling lasers, with both beams turning off simultaneously. The solid line is a numerical simulation of the density matrix with a single adjustable parameter. See text for details. 


\section{Localization experiment protocol}

We next discuss the details of the localization experiment. Observing nanoscale-level spatial localization is technically challenging for the following reasons: (i) Because the excitation is localized to spatial scales much smaller than the wavelength of light, the localized atoms cannot be imaged directly. (ii) The standing-wave pattern for the coupling laser must be spatially stable to within approximately $10 \mathrm{~nm}$ throughout the experiment in order to prevent the localization signal from washing out. To overcome these challenges, we have devised the following experimental protocol. In the spirit of the autocorrelation technique from ultrafast physics, we study localization with two EIT pulses that are separated by a controllable amount of time $\tau$. With the atoms starting in the ground level $F=1$, the first EIT pulse transfers atoms that are near the nodes of the standing wave to the $F=2$ level. We then turn on a unidirectional beam, called the blow-away beam $\left(\Omega_{\text {blow-away }}\right)$, that is resonant with the $F=2 \rightarrow F^{\prime}=$ 3 cycling transition. This laser continually scatters photons only on atoms that have been transferred to the $F=2$ level, thus selectively removing them from the trap. We next spatially shift the standing-wave pattern for the coupling laser beam by a known amount, $\delta x$. This shift is achieved by adjusting the frequencies of the two counterpropagating coupling lasers so that they differ slightly. For a frequency difference of $\delta f$, the spatial shift of the standing-wave profile before the second EIT pulse is $\delta x=\delta f \tau(\lambda / 2)$. A second EIT pulse and a subsequent blow-away beam then repeats the process. Depending on how the standing-wave patterns during the first and the second EIT pulses overlap, we observe a change in the number of atoms that remain in the trap at the end of the experiment. If, for example, there is significant overlap between the patterns, the first EIT pulse-blow-away sequence would have already removed the atoms near the nodes from the trap, so the second would remove very few. However, if there is almost no overlap, the sequences would remove about equal numbers of atoms. In essence, we are "correlating" the localized excitation with itself to measure its spatial width. The variation in $\delta x$ can be accomplished by either changing the frequency difference between the two beams, $\delta f$, or varying the time delay between the two EIT pulses, $\tau$. In our experiments, we scan $\delta x$ by introducing a small frequency difference $\delta f$ between the two coupling lasers, and we keep the time delay between the two pulses fixed. The frequency difference $\delta f$ is kept constant throughout each experimental cycle. We note that for this procedure to work, the atoms must be cold enough such that the diffusion of atoms between EIT pulses does not wash out the excitation pattern due to the first EIT pulse.

Figure 5 shows the experimental timing protocol. We begin the experiment by loading the MOT for $1 \mathrm{~s}$. We turn on the dipole-trapping beam during the last $50 \mathrm{~ms}$ of the

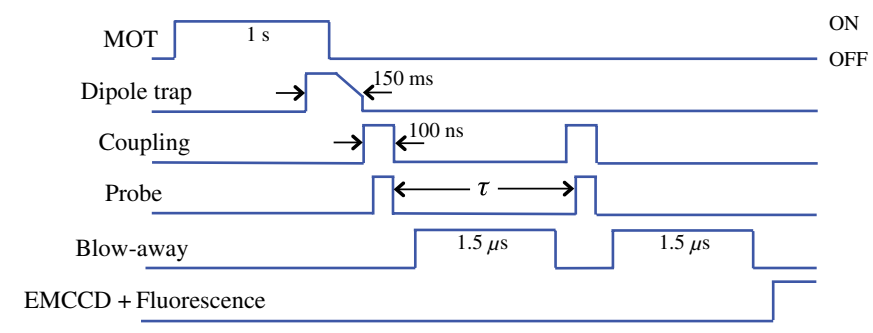

FIG. 5. The timing protocol for the localization experiment. In order to resolve nanometer spatial scales, we use two EIT pulses separated by $\tau$. The standing wave for the coupling laser is shifted to a different location between the two pulses. This shift is accomplished by setting the frequencies of the two counterpropagating beams to be slightly different. We obtain localization information through correlation measurements by scanning the spatial shift and recording the number of atoms that remain in the trap.

MOT loading cycle and transfer the atoms from the MOT to the dipole trap. The MOT magnetic field gradient is turned off during the dipole-trap loading interval, and the frequency and the intensity of the MOT lasers are adjusted to optimize the efficiency of loading. We also reduce the optical power in the hyperfine repumper beam for the MOT during the last few milliseconds of loading to increase the number of trapped atoms. The atoms are then evaporatively cooled to a temperature of $5.5 \mu \mathrm{K}$ by lowering the trap depth. We turn off the dipole-trap beam abruptly and perform the experiment $100 \mathrm{~ms}$ after the MOT beams are turned off. The dipole-trapping beam is turned off during the experiment to avoid the position-dependent AC Stark shifts of the transitions due to the trapping laser. At the start of the experiment, we first initialize all the atoms to the $F=1$ level using an appropriate optical pumping beam (not shown in Fig. 5). Each EIT pulse consists of a 100-nslong coupling laser pulse overlapped with a 50-ns-long probe pulse. As mentioned above, to adiabatically prepare the atoms into the dark state, the coupling laser is turned on before the probe laser beam. The probe and coupling laser pulses are turned off simultaneously. After each EIT pulse, we shine a $1.5-\mu$ s-long blow-away pulse to remove the atoms that have been transferred to the $F=2$ level.

\section{Localization experiment results}

Figure 6 shows the normalized number of atoms that remain in the trap as a function of the scanning distance of the standing wave. For these data, the probe power is held at a constant value of $5 \mathrm{~mW}$. In order to have effective EIT at all points along the standing wave, we introduce an intensity imbalance between the two coupling laser beams. As a result of the imbalance, there is no point with zero intensity, and the lowest intensity points of the standing wave have a coupling laser power of $3 \mathrm{~mW}$. This result guarantees optimal transfer near each low-intensity point of the standing-wave profile. The coupling laser power in 

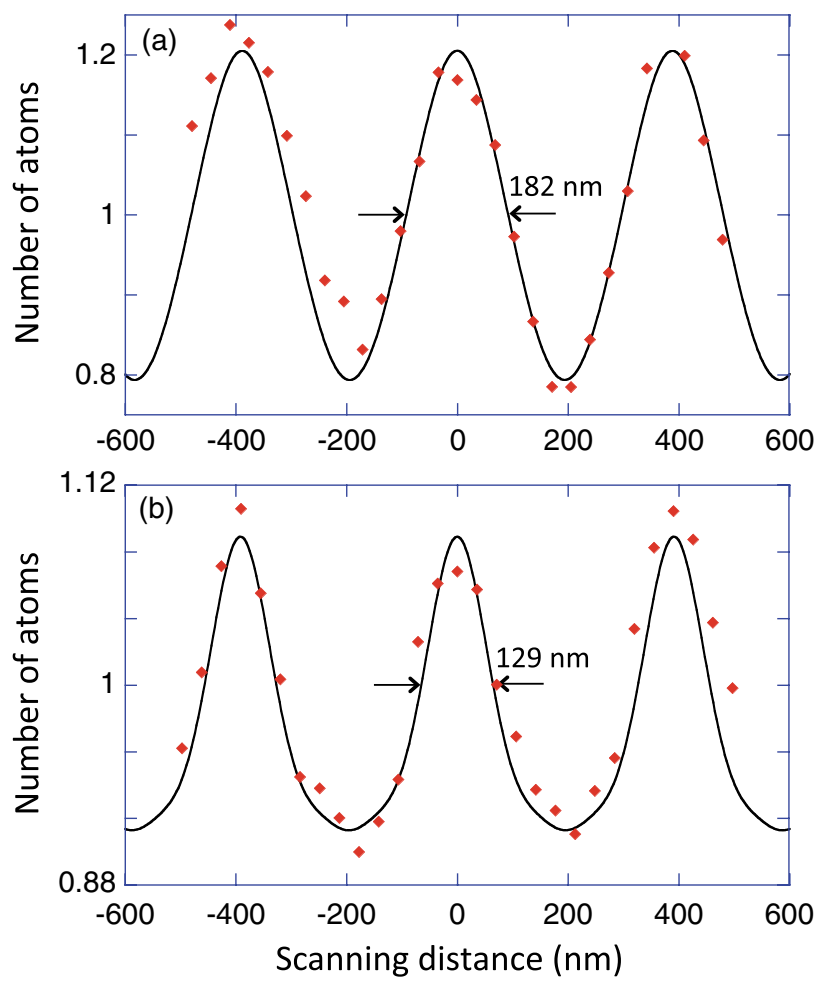

FIG. 6. The normalized number of atoms that remain in the trap as a function of the scanning distance of the standing wave. For these data, the power of the probe beam is held at a constant value of $5 \mathrm{~mW}$. The coupling laser power changes from $3 \mathrm{~mW}$ to $14 \mathrm{~mW}$ for part (a), and $3 \mathrm{~mW}$ to $30 \mathrm{~mW}$ for part (b). The solid lines are the results of numerical simulations, where we solve the density matrix equations at each point along the spatial profile of the standing wave. The simulations also take into account the diffusion of the atoms due to their finite temperature.

the standing wave changes from $3 \mathrm{~mW}$ to $14 \mathrm{~mW}$ for part (a), and $3 \mathrm{~mW}$ to $30 \mathrm{~mW}$ for part (b). As expected, the data are periodic, with a period that equals half the wavelength, $\lambda / 2=390 \mathrm{~nm}$. The width of the features becomes more localized as the peak intensity of the coupling laser is increased. The solid lines are the results of numerical simulations. With the exception of the slight spatial misalignment adjustment of the beams as discussed above, there are no adjustable parameters (i.e., all parameters in the simulations are experimentally measured). The simulations also take into account the diffusion of the atoms between the two EIT pulses due to their finite temperature. There is very good agreement with the simulation results and the experimental data. For the data of Fig. 6(b), the width of the features is $129 \mathrm{~nm}$, a value that is accurately reproduced by the numerical simulations.

Figure 7 shows the inferred population transfer along two cycles of the standing wave for the experimental conditions of Fig. 6(b). The transfer curve displayed in Fig. 7 is the result of the same simulation that produces good agreement with the experimental data of Fig. 6(b). The inferred width of the transfer is $100 \mathrm{~nm}$, which is a

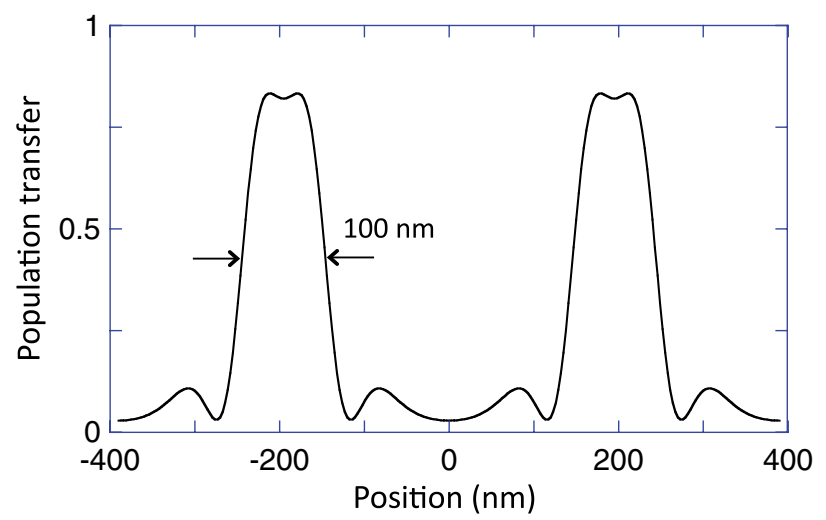

FIG. 7. The inferred population transfer from $F=1$ to $F=2$ along two cycles of the standing wave for the conditions of the experiment of Fig. 6(b). This transfer curve is the result of the same simulation that produces good agreement with the experimental data of Fig. 6(b). The inferred width of the transfer is $100 \mathrm{~nm}$, which is a factor of 8 smaller than the wavelength of the EIT laser beams.

factor of 8 smaller than the wavelength of the EIT laser beams. The shape and the width of the inferred transfer of Fig. 7 differ from the measurement of Fig. 6(b) for two key reasons: (i) the "correlation" effect of two spatial profiles and (ii) the diffusion of the atoms during the time interval $\tau$ due to their finite temperature. The observable correlation that we see in the experiment, shown in Fig. 6(b), is derived from the underlying unobservable spatial feature shown in Fig. 7 via convolution of that spatial feature with a delayed copy of itself. Because of the finite temperature of the atoms, one of the features blurs as the atoms move, while $\tau$ elapses between the EIT pulses.

Our numerical simulations predict that if the peak coupling laser power is further increased to values exceeding $100 \mathrm{~mW}$, then atomic localization below $40 \mathrm{~nm}$ should be observable. We have explored this high-power regime but have not been able to observe such tight localization. We believe that the limitations of our current experiments are as follows: (i) For high laser power, the coupling beam starts to interact with the nearby hyperfine levels in the excited electronic state. The frequency spacing between the $F^{\prime}=2$ and $F^{\prime}=3$ hyperfine levels in the $\mathrm{D}_{2}$ line is only $260 \mathrm{MHz}$ in ${ }^{87} \mathrm{Rb}$. As a result, the idealized three-level $\Lambda$ scheme approximation is no longer valid for high coupling laser intensities. (ii) For the correlation measurement to be a good indication of the excitation profile, we require the diffusion distance of the atoms due to their temperature to be smaller than the localization width. For an excitation width of $40 \mathrm{~nm}$, this requires atomic temperatures well below $1 \mu \mathrm{K}$ in the dipole trap. We will discuss below how we aim to overcome these limitations.

\section{CONCLUSIONS AND FUTURE DIRECTIONS}

In conclusion, we have reported an experiment in which the atomic excitation to a specific hyperfine level 
is localized to a spatial width of $100 \mathrm{~nm}$, a factor of 8 smaller than the wavelength of the laser beams used in the experiment. The localization is achieved using approximately 100-ns-long EIT laser pulses, i.e., with a gate speed capability of about $10 \mathrm{MHz}$. Our approach utilizes the dark state of EIT for atomic localization. As we discussed above, the dark-state-based approach has unique advantages that may be especially useful for quantum computing experiments in which nanoscale-level addressing with little decoherence is required.

In the future, our first immediate goal will be to overcome the limitations of the current experiments and to demonstrate atomic localization at the 10-nm level. For this purpose, we plan the following improvements: (i) We plan to switch to the $\mathrm{D}_{1}$ line excitation near a wavelength of $795 \mathrm{~nm}$. The use of the $\mathrm{D}_{1}$ line offers the following advantage over the $\mathrm{D}_{2}$ line excitation: The spacing between the hyperfine levels in $5 \mathrm{P}_{1 / 2}$ is $800 \mathrm{MHz}$, and as a result the undesired excitation into nearby levels is reduced. (ii) In our current experiment, the dipole-trapping laser is at a wavelength of $850 \mathrm{~nm}$, and as a result the photon scattering rate from the dipole-trapping laser is about $100 \mathrm{~Hz}$. Such a high photon scattering rate results in continuous heating of the atoms, which is quite detrimental for evaporative cooling. We therefore plan to switch to a longer infrared wavelength for the dipole-trapping beam, which should allow longer evaporative cooling times. The goal will be to cool the atoms to well below $1 \mu \mathrm{K}$ in the dipole trap.

The next phase of this line of research would be to implement subwavelength-spatial-resolution single-qubit and two-qubit gates. As discussed in detail by Lukin and colleagues [31], the dark-state-based localization can be combined with additional coherent excitations to perform gates with nanometer spatial resolution.

\section{ACKNOWLEDGMENTS}

We thank Josh Weber and Dan Sikes for many helpful discussions. We also would like to thank the Air Force Office of Scientific Research (AFOSR), the Wisconsin Alumni Research Foundation (WARF), and the University of Wisconsin-Madison for financial support.

[1] M. A. Nielsen and I.L. Chuang, Quantum Computation and Quantum Information (Cambridge University Press, Cambridge, England, 2000).

[2] J. I. Cirac and P. Zoller, Quantum Computations with Cold Trapped Ions, Phys. Rev. Lett. 74, 4091 (1995).

[3] C. Monroe, D. M. Meekhof, B. E. King, W. M. Itano, and D. J. Wineland, Demonstration of a Fundamental Quantum Logic Gate, Phys. Rev. Lett. 75, 4714 (1995).

[4] L. Isenhower, E. Urban, X.L. Zhang, A. T. Gill, T. Henage, T. A. Johnson, T. G. Walker, and M. Saffman, Demonstration of a Neutral Atom Controlled-NOT Quantum Gate, Phys. Rev. Lett. 104, 010503 (2010).
[5] T. Wilk, A. Gaetan, C. Evellin, J. Wolters, Y. Miroshnychenko, P. Grangier, and A. Browaeys, Entanglement of Two Individual Neutral Atoms Using Rydberg Blockade, Phys. Rev. Lett. 104, 010502 (2010).

[6] M. O. Scully and M.S. Zubairy, Quantum Optics (Cambridge University Press, Cambridge, England, 1997).

[7] Stephen E. Harris, Electromagnetically Induced Transparency, Phys. Today 50, 36 (1997).

[8] O. Kocharovskaya, Amplification and Lasing without Inversion, Phys. Rep. 219, 175 (1992).

[9] M. Fleischhauer, A. Imamoglu, and J.P. Marangos, Electromagnetically Induced Transparency: Optics in Coherent Media, Rev. Mod. Phys. 77, 633 (2005).

[10] K. Bergmann, H. Theuer, and B.W. Shore, Coherent Population Transfer among Quantum States of Atoms and Molecules, Rev. Mod. Phys. 70, 1003 (1998).

[11] J. Gea-Banacloche, Y. Li, S. Jin, and M. Xiao, Electromagnetically Induced Transparency in Laddertype Inhomogeneously Broadened Media: Theory and Experiment, Phys. Rev. A 51, 576 (1995).

[12] A. Kasapi, M. Jain, G. Y. Yin, and S.E. Harris, Electromagnetically Induced Transparency: Propagation Dynamics, Phys. Rev. Lett. 74, 2447 (1995).

[13] M. Xiao, Y. Li, S. Jin, and J. Gea-Banacloche, Measurement of Dispersive Properties of Electromagnetically Induced Transparency in Rubidium Atoms, Phys. Rev. Lett. 74, 666 (1995).

[14] L. V. Hau, S.E. Harris, Z. Dutton, and C.H. Behroozi, Light Speed Reduction to $17 \mathrm{~m} / \mathrm{s}$ in an Ultracold Atomic Gas, Nature (London) 397, 594 (1999).

[15] M. M. Kash, V. A. Sautenkov, A. S. Zibrov, L. Hollberg, G. R. Welch, M. D. Lukin, Y. Rostovtsev, E. S. Fry, and M. O. Scully, Ultraslow Group Velocity and Enhanced Nonlinear Effects in a Coherently Driven Atomic Gas, Phys. Rev. Lett. 82, 5229 (1999).

[16] Y. Rostovtsev, O. Kocharovskaya, G. R. Welch, and M. O. Scully, Slow, Ultraslow, Stored, and Frozen Light, Opt. Photonics News 13, 44 (2002).

[17] G. M. Gehring, A. Schweinsberg, C. Barsi, N. Kostinski, and R.W. Boyd, Observation of Backward Pulse Propagation through a Medium with a Negative Group Velocity, Science 312, 895 (2006).

[18] M. Fleischhauer and M. D. Lukin, Dark State Polaritons in Electromagnetically Induced Transparency, Phys. Rev. Lett. 84, 5094 (2000).

[19] D. F. Phillips, A. Fleischhauer, A. Mair, R. L. Walsworth, and M. D. Lukin, Storage of Light in Atomic Vapor, Phys. Rev. Lett. 86, 783 (2001).

[20] C. Liu, Z. Dutton, C.H. Behroozi, and L.V. Hau, Observation of Coherent Information Storage in an Atomic Medium Using Halted Light Pulses, Nature (London) 409, 490 (2001).

[21] I. Novikova, A. V. Gorshkov, D. F. Phillips, A. S. Sorensen, M. D. Lukin, and R. L. Walsworth, Optimal Control of Light Storage and Retrieval, Phys. Rev. Lett. 98, 243602 (2007).

[22] N. B. Phillips, A. V. Gorshkov, and I. Novikova, Optimal Light Storage in Atomic Vapor, Phys. Rev. A 78, 023801 (2008).

[23] H. Schmidt and A. Imamoglu, Giant Kerr Nonlinearities Obtained by Electromagnetically Induced Transparency, Opt. Lett. 21, 1936 (1996). 
[24] A. Imamoglu, H. Schmidt, G. Woods, and M. Deutsch, Strongly Interacting Photons in a Nonlinear Cavity, Phys. Rev. Lett. 79, 1467 (1997).

[25] S.E. Harris and Y. Yamamoto, Photon Switching by Quantum Interference, Phys. Rev. Lett. 81, 3611 (1998).

[26] M. D. Lukin and A. Imamoglu, Nonlinear Optics and Quantum Entanglement of Ultraslow Photons, Phys. Rev. Lett. 84, 1419 (2000).

[27] H. Wang, D. Goorskey, and M. Xiao, Enhanced Kerr Nonlinearity via Atomic Coherence in a Three-Level Atomic System, Phys. Rev. Lett. 87, 073601 (2001)

[28] H. Kang and Y. Zhu, Observation of Large Kerr Nonlinearity at Low Light Intensities, Phys. Rev. Lett. 91, 093601 (2003).

[29] G.S. Agarwal and K. T. Kapale, Subwavelength Atom Localization via Coherent Population Trapping, J. Phys. B 39, 3437 (2006).

[30] D. D. Yavuz and N.A. Proite, Nanoscale Resolution Fluorescence Microscopy Using Electromagnetically Induced Transparency, Phys. Rev. A 76, 041802(R) (2007).

[31] A. V. Gorshkov, L. Jiang, M. Greiner, P. Zoller, and M. D. Lukin, Coherent Quantum Optical Control with Subwavelength Resolution, Phys. Rev. Lett. 100, 093005 (2008).

[32] H. Li, V. A. Sautenkov, M. M. Kash, A. V. Sokolov, G. R. Welch, Y. V. Rostovtsev, M. S. Zubairy, and M. O. Scully, Optical Imaging Beyond the Diffraction Limit via Dark States, Phys. Rev. A 78, 013803 (2008).

[33] N. A. Proite, Z. J. Simmons, and D. D. Yavuz, Observation of Atomic Localization Using Electromagnetically Induced Transparency, Phys. Rev. A 83, 041803(R) (2011).

[34] J.E. Thomas, Uncertainty Limited Position Measurement of Moving Atoms Using Optical Fields, Opt. Lett. 14, 1186 (1989).

[35] J.E. Thomas, Quantum Theory of Atomic Position Measurement Using Optical Fields, Phys. Rev. A 42, 5652 (1990).

[36] K. D. Stokes, C. Schnurr, J. R. Gardner, M. Marable, G. R. Welsch, and J.E. Thomas, Precision Position Measurement of Moving Atoms Using Optical Fields, Phys. Rev. Lett. 67, 1997 (1991).

[37] J. R. Gardner, M.L. Marable, G. R. Welsch, and J.E. Thomas, Suboptical Wavelength Position Measurement of Moving Atoms Using Optical Fields, Phys. Rev. Lett. 70, 3404 (1993).

[38] F. Le Kien, G. Rempe, W. P. Schleich, and M. S. Zubairy, Atom Localization via Ramsey Interferometry: A Coherent Cavity Field Provides a Better Resolution, Phys. Rev. A 56, 2972 (1997).

[39] S. Qamar, S. Y.Zhu, and M. S.Zubairy, Atom Localization via Resonance Fluorescence, Phys. Rev. A 61, 063806 (2000).

[40] K. T. Kapale, S. Qamar, and M. S. Zubairy, Spectroscopic Measurement of an Atomic Wave Function, Phys. Rev. A 67, 023805 (2003).
[41] M. Sahrai, H. Tajalli, K. T. Kapale, and M. S. Zubairy, Subwavelength Atom Localization via Amplitude and Phase Control of the Absorption Spectrum, Phys. Rev. A 72, 013820 (2005).

[42] M. Macovei, J. Evers, C. H. Keitel, and M. S. Zubairy, Localization of Atomic Ensembles via Superfluorescence, Phys. Rev. A 75, 033801 (2007).

[43] J. T. Chang, J. Evers, M. O. Scully, and M. S. Zubairy, Measurement of the Separation Between Atoms Beyond Diffraction Limit, Phys. Rev. A 73, 031803(R) (2006).

[44] E. Paspalakis and P.L. Knight, Localizing an Atom via Quantum Interference, Phys. Rev. A 63, 065802 (2001).

[45] J. Xu and X. Hu, Sub-half-wavelength Localization of an Atom via Trichromatic Phase Control, J. Phys. B 40, 1451 (2007).

[46] P. Storey, M. Collett, and D. Walls, Measurement Induced Diffraction and Interference of Atoms, Phys. Rev. Lett. 68, 472 (1992).

[47] R. Quadt, M. Collett, and D. Walls, Measurement of Atomic Motion in a Standing Light Field by Homodyne Detection, Phys. Rev. Lett. 74, 351 (1995).

[48] S. Kunze, K. Dieckmann, and G. Rempe, Diffraction of Atoms from a Measurement Induced Grating, Phys. Rev. Lett. 78, 2038 (1997).

[49] M. Holland, S. Marksteiner, P. Marte, and P. Zoller, Measurement Induced Localization from Spontaneous Decay, Phys. Rev. Lett. 76, 3683 (1996).

[50] J. Mompart, V. Ahufinger, and G. Birkl, Coherent Patterning of Matter Waves with Subwavelength Localization, Phys. Rev. A 79, 053638 (2009).

[51] S. W. Hell, Far-Field Optical Nanoscopy, Science 316, 1153 (2007).

[52] P. C. Maurer, J. R. Maze, P. L. Stanwix, L. Jiang, A. V. Gorshkov, A. A. Zibrov, B. Harke, J.S. Hodges, A. S. Zibrov, A. Yacoby, D. Twitchen, S.W. Hell, R. L. Walsworth, and M. D. Lukin, Far-Field Optical Imaging and Manipulation of Individual Spins with Nanoscale Resolution, Nat. Phys. 6, 912 (2010).

[53] D. Viscor, J. L. Rubio, G. Birkl, J. Mompart, and V. Ahufinger, Single-Site Addressing of Ultracold Atoms Beyond the Diffraction Limit via Position-Dependent Adiabatic Passage, Phys. Rev. A 86, 063409 (2012).

[54] Q. Sun, J. Evers, M. Kiffner, and M. S. Zubairy, Subwavelength Optical Lattices Induced by PositionDependent Dark States, Phys. Rev. A 83, 053412 (2011).

[55] M. Kiffner, J. Evers, and M.S. Zubairy, Resonant Interferometric Lithography Beyond the Diffraction Limit, Phys. Rev. Lett. 100, 073602 (2008).

[56] N. A. Proite, B.E. Unks, J. T. Green, and D. D. Yavuz, Refractive Index Enhancement with Vanishing Absorption in an Atomic Vapor, Phys. Rev. Lett. 101, 147401 (2008).

[57] B. E. Unks, N. A. Proite, and D. D. Yavuz, Generation of Laser Light with GHz Splitting, Rev. Sci. Instrum. 78, 083108 (2007). 